\title{
MONITORING AND POSITIONING METHOD OF INDOOR MOBILE ROBOT USING WIRELESS NETWORK
}

\author{
Luna Fan Quoc 1 , Tien Le ${ }^{2}$, Shahreen Kasim³ \\ ${ }^{1}$ Henan Vocational College of Art, Cultural Communication Technology Department \\ Henan Zhengzhou, 450002, China \\ ${ }^{2}$ Haiphong Department of Education and Training, Haiphong, Vietnam \\ ${ }^{3}$ Faculty of Computer Science and Information Technology, Universiti Tun Hussein Onn Malaysia \\ Email: lunafan43912@163.com
}

\begin{abstract}
The objective of this study is to realize the monitoring, positioning and route planning of indoor mobile robot, so that the brain of indoor mobile robot can form the route planning map in different environments, and finally achieve the best route navigation of indoor mobile robot. In this study, the ultra-wideband (UWB) wireless network location method of indoor mobile robot is proposed with laser pulse ranging method to measure the positioning distance of indoor mobile robots. Secondly, the trilateral positioning method is used to measure the position of the starting node to several known ending nodes, to estimate the position of the starting node of the indoor mobile robot. Finally, gyroscopes and odometers are used to measure the rectangular and U-shaped motion of indoor mobile robots. The results show that the sampling frequency of $50 \mathrm{~Hz} \mathrm{UWB}$ wireless network is used to collect offline information about the mobile indoor robot. After the information data is processed, the measurement noise covariance matrix can be obtained, and finally, the expected target can be obtained, and the positioning distance measurement of indoor mobile robot can be realized. When the robot glides along the rectangular road with a starting point of $(2.48,2.15)$ and the U-shaped road with a starting point of $(7.16,6.31)$ at a constant speed, its UWB measurement values basically jump near the route. At the same time, there will be noise, and the density of the numerical results is relatively low, which means that the UWB can find a stable direction for the indoor mobile robot, but it is unable to measure the path angle deviation of the robot. The deviation angles of the robot rectangular route are $90^{\circ}, 180^{\circ}, 270^{\circ}$ and $360^{\circ}$ respectively. The deviation angle error of gyroscope will increase with the increase of robot moving time. The deviation angle errors of the rectangular and U-shaped routes measured by the odometer are kept around the 0 reference line, which indicates that compared with the gyroscope, the odometer can correct the angle by the specific calculation method when the angle deviation is large, so as to keep the error around the 0 reference line. The results can provide a practical basis for indoor mobile robot to monitor and locate accurately through wireless network.
\end{abstract}

Keywords: Indoor Mobile Robot, UWB Wireless Network, Motion State, Indoor Positioning.

\section{Introduction}

With the development of science and technology, artificial intelligence technology [1] has gradually developed in various fields, constantly changing human life. As the result of artificial intelligence technology, robot [2] is gradually developed and applied by all countries in the world, and progressively enters the families of ordinary people, bringing convenience and superior performance to the lives. However, with economic growth and the improvement of people's speaking level, the performance requirements of robots are higher and higher, which forces the research of robots to a higher level.

For the requirements of machine performance, it is the most common and core problem to be able to move autonomously, and the working environment of autonomous mobile robot is generally a complex indoor place. Therefore, the research on the performance of indoor mobile robot is the focus at home and abroad. In the foreign research on indoor mobile robot, it has been able to use its own sensor equipment to obtain and analyze the surrounding environment information, and the map obtained through analysis can determine its location, so as to carry out positioning and navigation [3]. Although the research on indoor mobile robot started late in China, it has made rapid progress. At present, deep learning [4] has been integrated into the vision navigation of indoor mobile robot, and neural network [5-6] has been applied to the actual movement environment of indoor mobile robot. After repeated training, the robot can adapt to the 
new indoor environment, so as to achieve the ability level of human expectations.

In this study, firstly, the indoor location method based on wireless network is proposed, and the specific TagNode of indoor mobile robot is obtained by using TOF two-way ranging technology and trilateration location algorithm. Then, an optimized algorithm combining wireless network and coding equipment is proposed to improve the positioning accuracy of indoor robot. Through the indoor robot experiment, it is proved that the accuracy of the above algorithm can improve the positioning accuracy and position estimation of indoor mobile robot as well as can provide a feasible basis for the performance mining of indoor robot.

\section{Method}

\subsection{Indoor location method based on wireless network}

General indoor wireless network positioning methods include infrared positioning, WiFi Positioning and ultra-wideband (UWB) positioning [7]. The characteristics of mobile positioning of indoor robots are shown in the table below:

Table 1. Characteristics of indoor wireless network positioning methods

\begin{tabular}{|c|c|c|c|c|}
\hline Method & Accuracy (m) & Applicable area (m) & Price & Complexity \\
\hline Infrared positioning & {$[6,10]$} & {$[2,6]$} & $\begin{array}{c}\text { medium and high } \\
\text { grades }\end{array}$ & weak \\
\hline WIFI positioning & {$[2,10]$} & {$[21,49]$} & $\begin{array}{c}\text { low and medium } \\
\text { grade }\end{array}$ & weak \\
\hline UWB positioning & {$[0.1,3]$} & {$[2,50]$} & mid-grade & middle \\
\hline $\begin{array}{c}\text { Bluetooth } \\
\text { positioning }\end{array}$ & {$[2,9]$} & {$[2,29]$} & low-grade & weak \\
\hline
\end{tabular}

UWB positioning method is a relatively cutting-edge wireless network technology. Its total broadband frequency exceeds $500 \mathrm{MHz}$, and the duration of frequency signal is very short. Therefore, the positioning method has strong time discrimination, which determines that the absolute advantage of UWB positioning method over other positioning methods is that it has a high indoor positioning accuracy [8-9]. Considering the price and performance, UWB indoor wireless positioning method is used in the research of indoor mobile robot monitoring and positioning.

First, the mechanism of UWB location method. This method was first used in military operations, and then used in the field of wireless technology. Because of its wide bandwidth, it is not easy to be interfered by clutter in wireless communication, and the communication effect is very good [10].

The calculation method of the relative frequency bandwidth in the UWB spectrum is the ratio of the frequency bandwidth of the signal to the core frequency, as shown in the following equation:

$$
D=\frac{2 \times\left(f_{u}-f_{m}\right)}{f_{u}+f_{m}}
$$

In the equation, $f_{u}$ and $f_{m}$ represent the corresponding maximum and minimum frequency values when the peak power is reduced by $8 \mathrm{~dB}$.

Generally, the relative width of the narrow frequency band is not more than 0.01 , the relative width ratio of the wide frequency band is between $0.01-0.2$, and the relative width ratio of the ultrawide frequency band is more than 0.02 .
Secondly, the advantages of UWB over other wireless location methods are as follows.

First, it contains a large amount of information and is fast. In the information channel with white noise, the maximum zero error transmission speed that UWB can achieve is as follows:

$$
D=A \times \log _{2}(1+K E P)
$$

In the equation, $A$ is the width of the frequency band and KEP is the signal-to-noise ratio. According to the frequency bandwidth of UWB, it shows that under the strong noise environment, the communication of UWB can achieve the transmission efficiency of more than 100 megabytes, and it is also more suitable for the equipment that needs highspeed transmission of information.

Second, it has high confidentiality protocol. The radiation spectral density of UWB is very low compared with other wireless networks, which is generally lower than the noise in the background environment. Compared with other narrow frequency, UWB's own frequency signal does not produce noise interference, so UWB can exist together with other narrow frequency bands. At the same time, due to its low spectral density, it is not easy to be intercepted by other devices in the range, so it has high security performance.

Third, the accuracy of location and distance measurement is high. UWB has a high resolution in space and time dimensions, therefore its refresh rate is very high. Hence, UWB will have a strong performance in location and distance measurement. 


\subsection{Laser pulse ranging}

The use of wireless networks for information synchronization requires specific algorithms to achieve. Under the condition of ensuring positioning accuracy and time synchronization, it is also necessary to ensure that a wireless network system is easy to be developed and operated. To solve the mentioned problems, in this study, the laser pulse ranging method [11-12] is used to measure the positioning distance of indoor mobile robot. Consequently, travel distance of the robot is determined by the time consumed between the starting point of the laser pulse signal and the stop node, to lay the foundation for determining the orientation of the robot. The schematic diagram of distance measurement from the starting point of the marker to the stop node is as follows:

The upper part of the figure represents the time stamp of the mark start point and the lower side represents the time stamp of the stop node. After a certain period of time, the marked nodes will carry out the same distance measurement. At the same time, the initial measurement node will monitor all the time, and once receiving the distance measurement feedback information of the marked node, it will respond. Thus, the back and forth time between the start node and the end node can be calculated, and finally the measured results can be transmitted to the start node [13].

For the starting node, the error time of transmitting information back and forth is as follows:

$$
T_{Y}=T_{Z}-T_{S}
$$

For the end node, the error time is as follows:

$$
T_{U}=T_{Q}-T_{W}
$$

The response consumption time of the starting node is expressed in equation 5 :

$$
T_{E}=T_{R}-T_{I}
$$

The response consumption time of the end node is as follows:

$$
T_{o}=T_{p}-T_{A}
$$

The total error time in the process of conducting the signal of laser pulse ranging refers to the time from input to output response of node plus the conducting time when the signal is in the air [14-15]. The actual flight time of signal transmission needs to remove the response consumption time of the start node and the end node. The calculation equation is as follows:

$$
T_{K}=\frac{T_{Y}+T_{U}+T_{E}+T_{O}}{4}
$$

From the above equation, the distance between the start node and the end node can be calculated as follows:

$$
D=c \times T_{K}
$$

In above equation, $c$ represents the speed of light.

\subsection{Trilateration positioning method}

The distance between the start node, and the end node is measured by the conducted signal of laser pulse ranging. According to the above distance and geometric calculation, the specific location of the label can be obtained. If the location of the start node is located, it is necessary to know at least three end nodes. The installation point of the end node is generally located at a higher position, and the start node is installed on the robot with a lower potential, so as to avoid the end node and the start node being blocked. When the above requirements are met, the start node and the end node are not in the same plane, so it is necessary to map the measured data to the same plane.

If the distance between the start node and each end node is $d_{1}, d_{2}, \ldots d_{m}$, the directions of each end node are $\left(a_{1}, b_{1}\right),\left(a_{1}, b_{2}\right), \ldots\left(a_{m}, b_{m}\right)$, the height of each node from the ground is $l_{1}, l_{2}, \ldots l_{m}$, the height of the starting node from the ground is $l_{0}$, according to Pythagorean theorem, the distance calculation equation mapped to the n-th end node on the plane is as follows:

$$
h_{n}=\sqrt{\left(d_{n}^{2}-\left(l_{\mathrm{n}}-l_{0}\right)^{2}\right)} \quad \mathrm{n}=1,2, \ldots, \mathrm{m}
$$

If the distance in the same plane is calculated, the trilateration positioning measurement method [1617] can be used for node positioning.

Trilateral positioning measurement is a geometric method, which measures the position from the start node to several known end nodes, to estimate the position of the start node. If the starting point orientation is (c, d) and is the orientation to be calculated, the equation to be solved is as follows:

$$
\left\{\begin{array}{c}
h_{1}^{2}=\left(c-c_{1}\right)^{2}+\left(d-d_{1}\right)^{2} \\
h_{2}^{2}=\left(c-c_{2}\right)^{2}+\left(d-d_{2}\right)^{2} \\
\mathrm{M} \\
h_{m}^{2}=\left(c-c_{m}\right)^{2}+\left(d-d_{m}\right)^{2}
\end{array}\right.
$$

By substituting and subtracting the equation 9 from the above equation we get: 


$$
\left\{\begin{array}{l}
h_{1}^{2}-h_{m}^{2}=c_{1}^{2}-c_{m}^{2}+d_{1}^{2}-d_{m}^{2}-2 c\left(c_{1}-c_{m}\right)-2 d\left(d_{1}-d_{m}\right) \\
h_{2}^{2}-h_{m}^{2}=c_{2}^{2}-c_{m}^{2}+d_{2}^{2}-d_{m}^{2}-2 c\left(c_{2}-c_{m}\right)-2 d\left(d_{2}-d_{m}\right) \\
\quad \mathrm{M} \\
h_{m-1}^{2}-h_{m}^{2}=c_{m-1}^{2}-c_{m}^{2}+d_{m-1}^{2}-d_{m}^{2}-2 c\left(c_{m-1}-c_{m}\right)-2 d\left(d_{m-1}-d_{m}\right)
\end{array}\right.
$$

Equation (11) can be expressed in the form of below equation (12):

$$
E\left[\begin{array}{l}
x \\
y
\end{array}\right]=k
$$

Where,

$$
E=\left[\begin{array}{cc}
2\left(x_{1}-x_{m}\right) & 2\left(y_{1}-y_{m}\right) \\
2\left(x_{2}-x_{m}\right) & 2\left(y_{2}-y_{m}\right) \\
\mathrm{M} & \mathrm{M} \\
2\left(x_{m-1}-x_{m}\right) & 2\left(y_{m-1}-y_{m}\right)
\end{array}\right], k=\left[\begin{array}{cc}
x_{1}^{2}-x_{m}^{2}+y_{1}^{2}-y_{m}^{2}-k_{1}^{2}+k_{m}^{2} \\
x_{2}^{2}-x_{m}^{2}+y_{2}^{2}-y_{m}^{2}-k_{2}^{2}+k_{m}^{2} \\
\mathrm{M} & \mathrm{M} \\
x_{m-1}^{2}-x_{m}^{2}+y_{m-1}^{2}-y_{m}^{2}-k_{m-1}^{2}+k_{m}^{2}
\end{array}\right]
$$

According to the above equation, the orientation of the starting node is calculated as follows:

$$
\left[\begin{array}{l}
x \\
y
\end{array}\right]=E^{-1} k
$$

\subsection{Motion state measurement of indoor mobile robot}

Firstly, the state equation of robot motion is described. In the plane, the starting node can be used to obtain the orientation coordinates $(x, y)$ of the indoor mobile robot. The linear velocity $v$ of mobile robot can be measured by odometer. The gyroscope can be used to measure the yaw rate $\delta$ of the mobile robot. The model state of mobile robot at time i can be expressed as follows:

$$
U_{i}=\left[\begin{array}{lllll}
x_{i} & \mathrm{y}_{\mathrm{i}} & \delta_{\mathrm{i}} & \mathrm{v}_{\mathrm{i}} & \stackrel{\mathrm{g}}{\delta}
\end{array}\right]^{P}
$$

The equation of motion state of the robot is as follows:

$$
\hat{U}_{i}^{-}=j\left(\hat{U}_{i-1}^{\Lambda}, O_{i-1}, \mathrm{r}_{i-1}\right)
$$

Among them, $\stackrel{\Lambda}{U_{i-1}}$ represents the posterior estimation of time, $U_{i}^{-}$represents the prior estimation, $O_{\mathrm{i}-1}$ represents the control value of $\mathrm{i}-1$ time, ri-1 represents the noise value of $\mathrm{i}-1$ time. The control value of robot model $\mathrm{Oi}=0$.

Secondly, the measurement equation of the robot is described. The azimuth information $(a, b)$ are measured by the laser pulse ranging conducted signal, the velocity $v$ is measured by the odometer, and the angular velocity $\delta$ is measured by the gyroscope. Following equation was used for measurement:

$$
F_{\mathrm{i}}=G C_{i}+\alpha_{i}
$$

In the equation, $F_{\mathrm{i}}=\left[\begin{array}{c}x \\ y \\ v \\ \delta\end{array}\right]_{i}, \quad G=\left[\begin{array}{lllll}1 & 0 & 0 & 0 & 0 \\ 0 & 0 & 1 & 0 & 0 \\ 0 & 0 & 0 & 0 & 1 \\ 0 & 0 & 0 & 0 & 1\end{array}\right], \quad \alpha_{\mathrm{i}}$ represents noise.

\section{Result}

The sampling frequency of $50 \mathrm{~Hz}$ is used to collect the off-line information of the mobile indoor robot. After the information data is processed, the measurement noise covariance matrix $P_{n}$ can be obtained. The value of the process noise variance matrix $Y_{h}$ is adjusted until the filter meets the expected target. The results of $P_{n}$ and $Y_{h}$ are as follows:

$$
P_{n}=\left[\begin{array}{cccc}
\frac{1}{4} & 0 & 0 & 0 \\
0 & \frac{1}{4} & 1 & 0 \\
0 & 0 & \frac{1}{100} & 0 \\
0 & 0 & 0 & \frac{1}{25}
\end{array}\right], Y_{h}=\left[\begin{array}{ccccc}
\frac{1}{250} & 0 & 0 & 0 & 0 \\
0 & \frac{1}{250} & 1 & 0 & 0 \\
0 & 0 & \frac{1}{500} & 0 & 0 \\
0 & 0 & 0 & 0 & \frac{1}{650}
\end{array}\right]
$$

The accuracy of laser pulse ranging method to calculate the robot's orientation is verified. In this study, two mobile routes of indoor mobile robot are set up: rectangular and U-shaped.

\subsection{Rectangular motion route monitoring and positioning of indoor mobile robot}

The robot glides at a constant speed along Route 1 , starting from $(2.48,2.15)$, and then from route: $a$, $\mathrm{b}, \mathrm{c}, \mathrm{d}$ to the end point, as shown in Figure 1 below: 


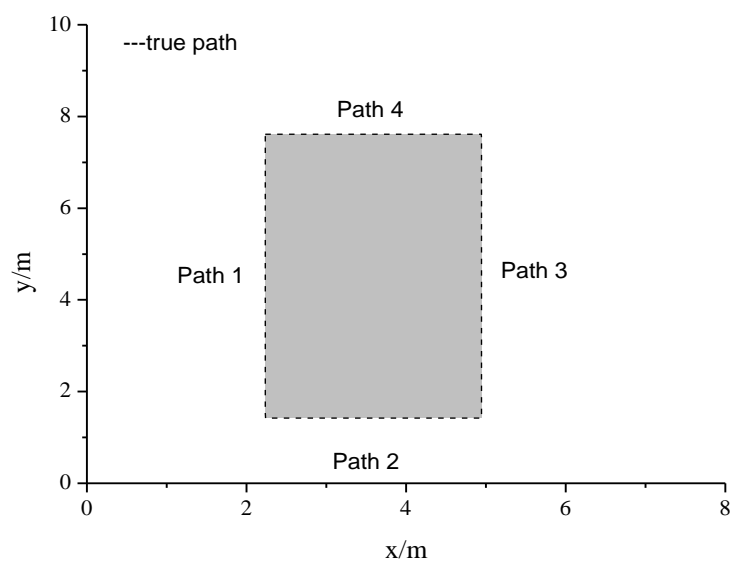

Figure 1: Square route path of indoor mobile robot

The measurement value (UWB) of the rectangular route laser pulse ranging of indoor mobile robot is shown in Figure 2 below:

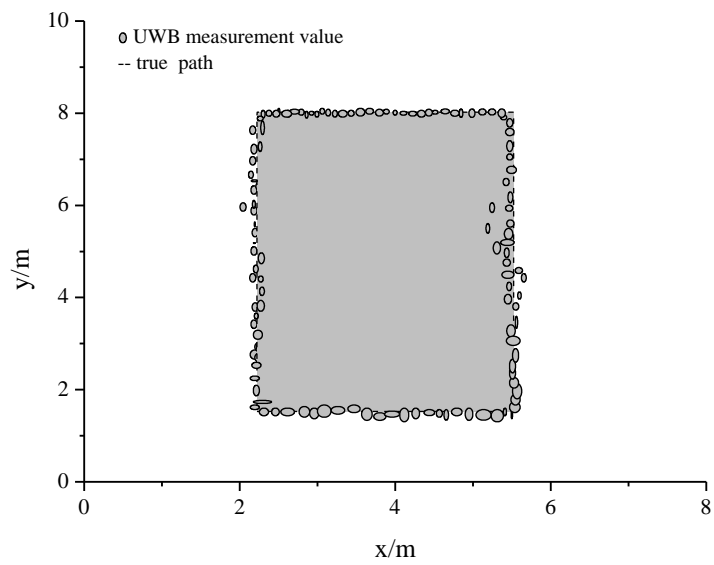

Figure 2: UWB measurement value of rectangular route of indoor mobile robot

As can be seen from the above figure, when the robot moves along the route in Figure 1, its UWB measurement values basically jump at the approach of the route, but there will be noise, and the density of the numerical results is relatively low, which means that UWB can find a stable orientation for the indoor mobile robot, but there is no way to measure the path angle deviation of the robot.

The indoor mobile robot is measured by gyroscope and odometer square track to obtain the position and attitude of the mobile robot, as shown in Figure 3 below:

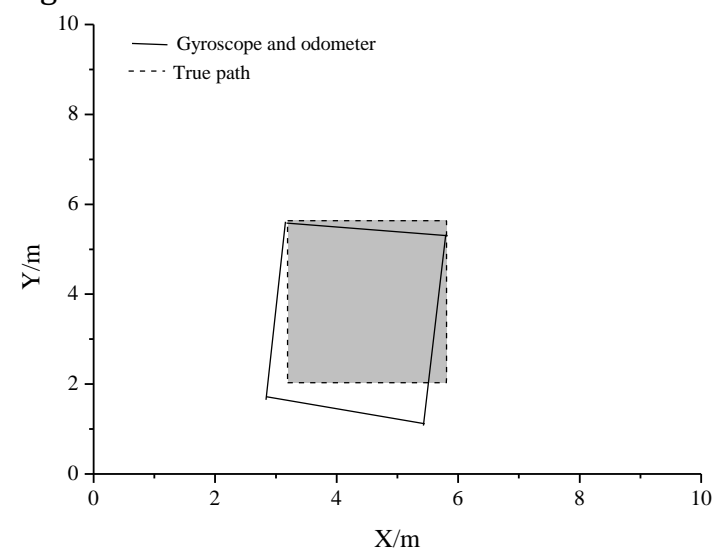

Figure 3: Measurement values of rectangular track gyroscope and odometer of mobile robot

As can be seen from the above figure, in a relatively short time, the route obtained by using gyroscope and odometer to measure the square trajectory of indoor mobile robot is more consistent with the real path. However, with the increase of time, the error of the path will gradually increase, and the indoor mobile robot will gradually deviate from the real path.

The deviation angles of robot moving from route 1 to route 4 are $90^{\circ}, 180^{\circ}, 270^{\circ}$ and $360^{\circ}$ respectively. Gyroscope and odometer are used to calculate the yaw angle of the robot when it moves. The error comparison between the two is shown in Table 2 and Figure 4 below:

Table 2. Angle deviation error comparison between gyroscope and odometer

\begin{tabular}{|c|c|c|c|c|c|}
\hline Route & $\begin{array}{c}\text { Position } \\
\text { measurement } \\
\text { method }\end{array}$ & Error Max & $\begin{array}{c}\text { Mean value of } \\
\text { error }\end{array}$ & $\begin{array}{c}\text { Mean square } \\
\text { error }\end{array}$ & $\begin{array}{c}\text { Standard } \\
\text { deviation }\end{array}$ \\
\hline \multirow{2}{*}{1} & gyroscope & 2.569900 & 1.253646 & 0.000477 & 0.019161 \\
& odometer & 10.55506 & 3.140048 & 0.0052605 & 0.049882 \\
\hline \multirow{2}{*}{2} & gyroscope & 3.150500 & 2.204350 & 0.001437 & 0.010465 \\
\cline { 2 - 3 } & odometer & 3.670263 & 1.570545 & 0.001522 & 0.021821 \\
\hline 3 & gyroscope & 5.104000 & 4.001874 & 0.005281 & 0.012600 \\
\cline { 2 - 3 } & odometer & 2.106513 & 0.503411 & 0.000241 & 0.015371 \\
\hline 4 & gyroscope & 7.051300 & 5.805703 & 0.010068 & 0.008446 \\
& & 6.675016 & 2.985011 & 0.004022 & 0.026501 \\
\hline
\end{tabular}

The angle deviation error curve of the rectangular track is shown as follows: 


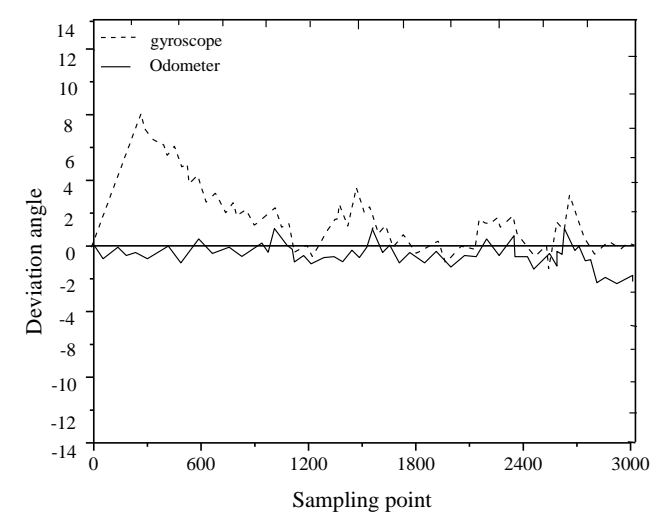

Figure 4: Deviation angle error curve of robot rectangular track

As can be seen from the above figure, the deviation angle error of gyroscope will increase with the increase of time. However, after using the odometer, the deviation angle errors of indoor mobile robots are kept around the zero-reference line. In the beginning, the deviation angle of gyroscope is relatively small, but the longer the time is, the error of angle deviation will continue to increase, which will not provide the indoor mobile robot with more correct numerical information. The odometer can correct the angle by a specific calculation method when the angle deviation is large, to keep the error around the zero-reference line.

\section{2 $\mathrm{U}$-shaped motion route monitoring and positioning of indoor mobile robot}

As the indoor mobile robot moves along the Ushaped route, the starting position of the robot is $(7.16,6.31)$, and it moves along the U-shaped route to the endpoint, as shown in Figure 5 below:

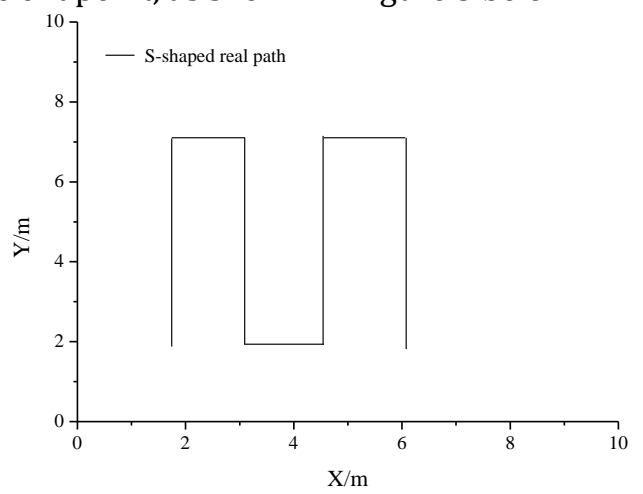

Figure 5: U-shaped mobile route of indoor mobile robot
The measurement value of U-shaped laser pulse ranging (UWB) of indoor mobile robot is shown in Figure 6 below:

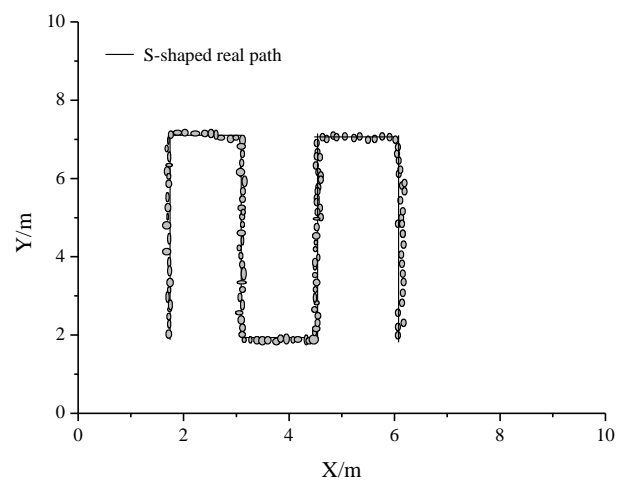

Figure 6: UWB measurement value of U-shaped route of indoor mobile robot

As can be seen from the above figure, when the robot moves along the route shown in Figure 5, its UWB measurement values are basically near the route but have a relatively high degree of dispersion.

The indoor mobile robot is measured by Ushaped trajectory of gyroscope and odometer to obtain the position and attitude of the mobile robot, as shown in Figure 7 below:

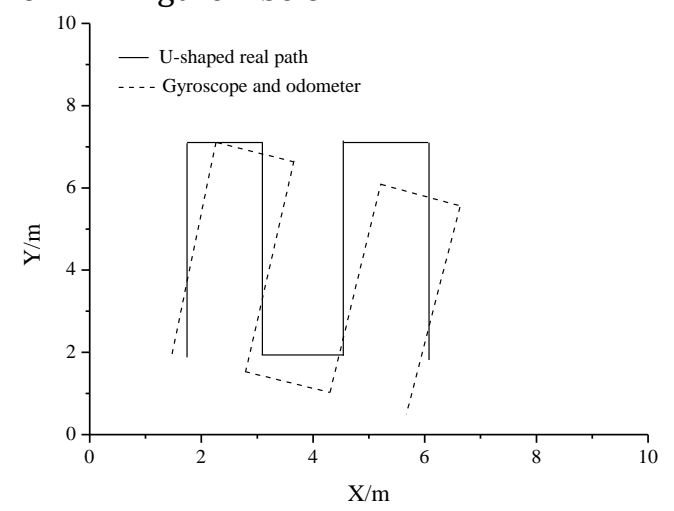

Figure 7: Measurement values of U-shaped trajectory gyroscope and odometer of mobile robot

As can be seen from the above figure, with the increase of time, the deviation of its path gradually increases, and the indoor mobile robot will gradually deviate from the real path.

The yaw angle of the robot is calculated by gyroscope and odometer. The error comparison between the two is shown in the following Table 3 and Figure 8:

Table 3. Comparison of angle deviation error between gyroscope and odometer

\begin{tabular}{|c|c|c|c|c|}
\hline Route & $\begin{array}{c}\text { Monitoring } \\
\text { method }\end{array}$ & Error (max) & Error (average) & SD \\
\hline \multirow{2}{*}{$\begin{array}{c}\text { U-shaped } \\
\text { trajectory }\end{array}$} & gyroscope & 13.991 & 11.872 & 0.0238 \\
\cline { 2 - 5 } & odometer & 7.188 & 3.987 & 0.0401 \\
\hline
\end{tabular}

The angle deviation error curve of U-shaped trajectory is shown in Figure 8 below: 


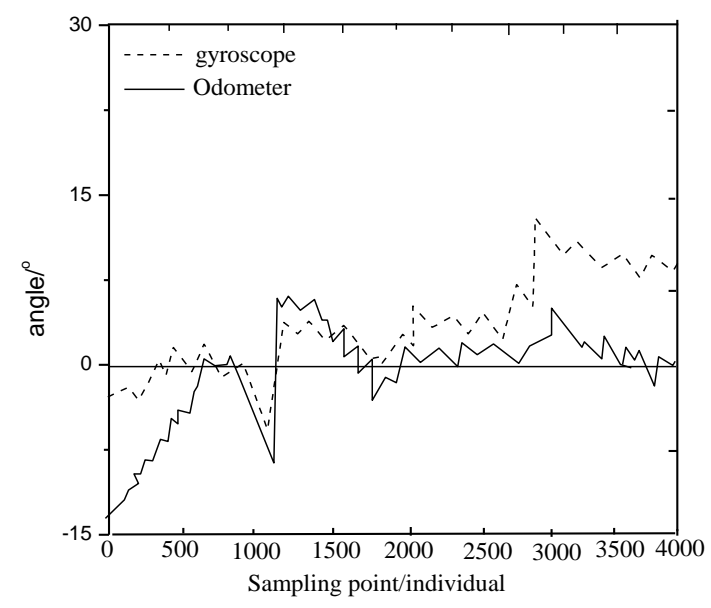

Figure 8: Deviation angle error curve of U-shaped trajectory of robot

It is clearly observable from above figure that according to the growth of time, the deviation angle error of gyroscope will also increase, but the deviation angle error of the indoor mobile robot will be kept around the zero-reference line after using odometer, which shows that the deviation angle of indoor mobile robot measured by odometer is relatively accurate.

\section{Discussion}

\subsection{Route planning of indoor mobile robot}

In this study, a rectangular mobile route map of indoor mobile robot is proposed, which is the most basic mobile route of the robot. When the robot moves along the square route, its UWB measurement values basically jump at the approach of the route, but there will be noise, and the density of the numerical results is relatively low, which shows that UWB can find a stable orientation for indoor mobile robot, which is the same as the positioning results of indoor mobile robot in the relevant literature that can move along the square route [18]. However, in this study, the relevant literature has not been referred to carry out other routes of indoor mobile robot mobile positioning, which is the problem to be solved in the follow-up research.

\subsection{Deviation angle of square route movement of indoor mobile robot}

In this study, two methods of measuring the deviation angle of the rectangular route of indoor mobile robot are proposed. Gyroscope and odometer can measure the deviation angle of the route of indoor mobile robot. However, with the increase of time, the deviation angle error of gyroscope will also increase, and the error of odometer in measuring the deviation angle of indoor mobile robot is basically kept around the zero-reference line.
The gyroscope has a small deviation angle at the beginning. However, with the increase of time, the error of angle deviation will continue to increase, which will not provide the indoor mobile robot with more correct numerical information. The odometer can correct the angle through a specific calculation method when the angle deviation is large, so as to keep the error around the zero-reference line, which is basically consistent with the results of indoor mobile robot route deviation angle measurement using the above methods in the relevant literature [19]. However, in this study, the relevant literature has not been referenced to measure the deviation angle of other routes of indoor mobile robot, which needs to be solved in follow-up research.

\section{Conclusions}

Indoor mobile robots carry out intelligent navigation. The core task is to locate and plan the target route according to its location. The current localization methods of indoor mobile robots are relatively simple, and the initial orientation of the robot must be given. At the same time, when the robot moves indoors, it will produce the phenomenon of repeated calculation of error, which greatly reduces the positioning accuracy of indoor mobile robot. UWB wireless network location is a new indoor wireless location method. Compared with other positioning methods, this positioning technology has the advantages of good traffic function, high positioning accuracy, and can successfully complete the monitoring and positioning of indoor mobile robot.

In this study, the positioning method of indoor mobile robot is studied. Based on the UWB wireless network, the indoor robot monitoring and positioning method are established. The measurement method of mobile deviation angle of indoor mobile robot is proposed, which is gyroscope and odometer. Compared with gyroscope, odometer can more accurately reflect the mobile orientation of indoor mobile robot and the angle of deviation from the route, which provides a feasible method for indoor mobile robot to accurately monitor and locate.

\section{References}

[1] Ignatov, Alexander, Ivchenko, Valeriy, Krug, Petr, et al. The Technologies for Remote Reconfiguration of Artificial Intelligence of Robotic Systems in Case of Mission or Driving Conditions Change. Applied Mechanics \& Materials, 2016, 851, pp. 477-483.

[2] Wei H, Chen Y H, Zhao Y. Adaptive Neural Network Control of an Uncertain Robot With Full-State Constraints. IEEE Transactions on Cybernetics, 2017, 46(3), pp. 620-629. 
[3] Zhe H, Zhu J G, Yang L H, et al. Accurate 3-D Position and Orientation Method for Indoor Mobile Robot Navigation Based on Photoelectric Scanning. IEEE Transactions on Instrumentation \& Measurement, 2015, 1(9), pp. 2518-2529.

[4] Li Z, Jia J D, Guan G, et al. Deep Learning based Improved Classification System for Designing Tomato Harvesting Robot. IEEE Access, 2018, (99), pp. 1-1.

[5] Wei H, Chen Y H, Zhao Y. Adaptive Neural Network Control of an Uncertain Robot With Full-State Constraints. IEEE Transactions on Cybernetics, 2017, 46(3), pp. 620-629.

[6] Abid Hossain Khan, Md Shafiqul Islam (2019). A Pctran-Based Investigation On The Effect of Inadvertent Control Rod Withdrawal On The Thermal-Hydraulic Parameters Of A Vver-1200 Nuclear Power Reactor. Acta Mechanica Malaysia, 2(2): 32-38, DOI: 10.26480/amm.02.2019.32.38

[7] Abdelkader T, Mohamed T, Abdelhalim S, et al. A Novel Design Method for Compact UWB Bandpass Filters. IEEE Microwave \& Wireless Components Letters, 2015, 25(1), pp. 4-6.

[8] Ardiansyah A, Nugraha G D, Hyojeong $H$, et al. A decision tree-based NLOS detection method for the UWB indoor location tracking accuracy improvement. International Journal of Communication Systems, 2019(4), pp. 3997.

[9] Ram Rohit Vannarth, K. Mallikharjuna Babu, P. Martin Jebraj (2019). Study On Sedimentation Stability of Mahua And Simarouba Oil Based Magnetorheological Fluids. Acta Mechanica Malaysia, 2(2): 39-44, DOI: 10.26480/amm.02.2019.39.44

[10] Huang S H, Yu G, Zha S H, et al. A Real-time Location System Based on RFID and UWB for Digital Manufacturing Workshop. Procedia Cirp, 2017, 63, pp. 132-137.
[11] Faiz Alam (2019). Laplace Adomian Decomposition Method For Solving A Model Of Chronic Myelogenous Leukemia ( $\mathrm{Cml}$ ) And $\mathrm{T}$ Cell Association. Matrix Science Mathematic, 3(2): 11-16

[12] Zhao Q H, Wang X Y, Bing H E, et al. Probability Model of Center-of-mass Calibration of Satellites' Retro-reflectors Used for Laser Ranging. Acta Geodaetica Et Cartographica Sinica, 2015, 44(4), pp. 370-376.

[13] Luo H J, Liu Q. Improved cumulative probabilities and range accuracy of a pulsed Geiger-mode avalanche photodiode laser ranging system with turbulence effects. Appl Opt, 2017, 56(29), pp. 8216-8223.

[14] Rohit Rana, Rajendra Kumar (2019). Performance Analysis of Aodv In Presence of Malicious Node. Acta Electronica Malaysia, 3(1): 01-05.

[15] Xu X B, Zhang H, Chen S S. Cramer-Rao lower bound on ranging precision of pulsed laser circular-viewing detection. Optik - International Journal for Light and Electron Optics, 2016, 127(22), pp. 10961-10970.

[16] Muller P, Peral-Rosado J D, Piche R, et al. Statistical Trilateration With Skew-t Distributed Errors in LTE Networks. 2016, 15(10), pp. 7114-7127.

[17] Ahmed Abugalia (2019). Effect of Corona on The Wave Propagation Along Overhead Transmission Lines. Acta Electronica Malaysia, 3(1): 06-09.

[18] Bruzzone L, Fanghella P. Functional Redesign of Mantis 2.0, a Hybrid Leg-Wheel Robot for Surveillance and Inspection. 2015, 81(2), pp. 116.

[19] Nithya N, Selvi D T. Human Cognition and Vision Based Earlier Path Determination System for Indoor Mobile Robot Path Planning. Advances in Intelligent Systems and Computing, 2015, 1089, pp. 727-732. 